\title{
Accuracy of STOP-Bang Questionnaire in Predicting Difficult Mask Ventilation: An Observational Study
}

\author{
Marium N. Khan ${ }^{1}$, Aliya Ahmed ${ }^{2}$ \\ 1. Anaesthetics, Blackpool Victoria Teaching Hospital, Blackpool, GBR 2. Anaesthesiology, Aga Khan University, \\ Karachi, PAK
}

Corresponding author: Aliya Ahmed, aliya.ahmed@aku.edu

\begin{abstract}
Introduction

Difficulty with bag-mask ventilation after the induction of general anesthesia and muscle relaxation places the patient at risk for a prolonged period of apnea and hypoxia and thus, at an increased risk of morbidity and mortality. This study was designed to assess the accuracy of the STOP-Bang questionnaire in predicting difficult mask ventilation (DMV) in patients receiving general anesthesia for elective surgical procedures.
\end{abstract}

\section{Methods}

It was a prospective cross-sectional, observational study conducted at a university teaching hospital. A total of 530 patients undergoing surgery under general anesthesia with endotracheal intubation were enrolled. STOP-Bang questionnaire was filled at pre-operative anesthesia assessment. Ease or difficulty of mask ventilation was assessed and documented by a senior resident responsible for intraoperative anesthetic management.

\section{Results}

Out of 530 patients, 139 (26.22\%) had a STOP-Bang score of $\geqslant 3$, of whom 55 (39.5\%) were found to have DMV. Out of 391 patients with a STOP-Bang score of $<3$, only 29 patients $(7.5 \%)$ had DMV ( $\mathrm{P} \leqslant 0.001)$. Snoring, high blood pressure, BMI more than $35 \mathrm{~kg} / \mathrm{m}^{2}$, age more than 50 years, neck circumference more than $40 \mathrm{~cm}$, and male gender were significantly associated with DMV. The accuracy of the STOP-Bang questionnaire in predicting difficult mask ventilation was $78.68 \%$ (95\% CI 74.99-81.95) with a negative predictive value of $92.58 \%$. The sensitivity and specificity were found to be $65.48 \%$ and $81.17 \%$ respectively.

\section{Conclusion}

STOP-Bang score has a high negative predictive value and can be very useful in ruling out the possibility of difficult mask ventilation.

Review began 06/13/2021 Review ended 06/15/2021 Published 06/27/2021

() Copyright 2021

Khan et al. This is an open access article distributed under the terms of the Creative Commons Attribution License CC-BY 4.0., which permits unrestricted use, distribution, and reproduction in any medium, provided the original author and source are credited.
Categories: Anesthesiology

Keywords: difficult mask ventilation, stop-bang score, difficult airway, general anesthesia, preoperative assessment

\section{Introduction}

Optimal oxygenation and ventilation of an anesthetized patient is the core responsibility of anesthesiologists and any difficulty in airway management is a major cause for concern. At the induction of anesthesia, neuromuscular blocking agents are commonly administered, which leads to muscle relaxation and apnea. The anesthesiologist performs mask ventilation for 3-4 minutes until the muscle relaxant takes its effect. Endotracheal intubation is then accomplished. Effective mask ventilation is therefore crucial for the initial 3-4 minutes before endotracheal intubation. Difficulties in mask ventilation place the patient at risk of prolonged apnea, with resulting hypoxia.

There are several factors that can predict possible difficulty in mask ventilation, obstructive sleep apnea (OSA) being one such factor [1]. Research has suggested that a high risk for OSA might be a predictor of difficulty in airway management during anesthesia [2-4]. However, patients suffering from OSA might be undiagnosed when they present for surgery $[1,5]$. The STOP-Bang questionnaire is a screening tool for assessing the risk for OSA [6,7]. Since it has been shown that OSA patients have a higher incidence of difficulty in mask ventilation $[2,8]$, it seems logical to assume that a high STOP-Bang score might be useful as an independent predictor of difficult mask ventilation.

A study on obese patients has shown that the STOP-Bang questionnaire is useful in predicting difficult intubation in these patients [8]. Identifying patients at an increased risk of OSA by assessing the STOP-Bang 
score during pre-anesthesia assessment and being prepared accordingly for possible difficulties in airway management with relevant armamentarium at hand would be useful in preventing adverse events related to difficult mask ventilation (DMV) and difficult endotracheal intubation [9]. Despite the globally growing recognition of the association of OSA with difficult airway management, available data about the relationship of high STOP-Bang score with DMV is scarce. In this study, we hypothesized that a high STOPBang score will be useful in predicting DMV and assessed the accuracy of the STOP-Bang questionnaire in predicting DMV in adult surgical patients.

\section{Materials And Methods}

Approval for the study was granted by the Ethics Review Committee of Aga Khan University (approval number: 4313-Ane-ERC-16). Patients included in the study comprised of those aged 18-60 years, having American Society of Anesthesiologists (ASA) status I-III, scheduled for elective surgery under general anesthesia and endotracheal intubation. Patients who refused to participate, those having neuromuscular disease, facial abnormalities and patients undergoing cardiothoracic, head and neck, obstetric, pediatric, neurosurgery, emergency surgery, or patients previously diagnosed with OSA (by polysomnography), having any airway related anatomical deformity (e.g. Down's syndrome) or pathological condition such as a history of head/neck surgery or radiation were excluded. Written informed consent was acquired from all study participants.

Eight easily administrable questions are included in the STOP-Bang questionnaire, each having a yes/no response (the questions are regarding snoring, tiredness or sleepiness during daytime, breath-holding during sleep, high blood pressure, body mass index (BMI), age, neck circumference and gender). A 'yes' response is given one mark and a 'no' represents zero. The marks are added up to obtain the final STOP-Bang score. The possible range of score is zero to eight.

The study participants were recruited from the scheduled elective surgical lists. The demographic data and STOP-Bang scores for each study participant who satisfied the inclusion criteria were recorded at the time of pre-anesthesia assessment by the resident assessing the patient. A score of $\geqslant 3$ was considered as an increased risk for OSA and $<3$ as having a low risk. Intra-operative observations were recorded in the study form by the anesthesia resident anesthetizing the patient under the supervision of a consultant anesthesiologist. It was ensured that the resident performing bag-mask ventilation on the study subjects had more than two years of anesthesia experience. The questionnaire and intra-operative study form were collected by the primary investigator from the residents. The anesthesiologist responsible for airway management was blinded to the STOP-Bang score.

Standard monitoring (electrocardiogram, non-invasive blood pressure, and peripheral oxygen saturation) was applied and baseline readings were noted. Pre-oxygenation was done for three minutes and induction of anesthesia was conducted with morphine $0.1 \mathrm{mg} / \mathrm{kg}$ and propofol $1-2 \mathrm{mg} / \mathrm{kg}$ until the patient became unresponsive to verbal communication, followed by the administration of atracurium $0.5 \mathrm{mg} / \mathrm{kg}$. Mask ventilation was then performed for three minutes using the circle system with the patient's head in a standard sniffing position. Mask ventilation was defined as easy when visible chest rise was achieved with the appearance of end-tidal carbon dioxide graph by a single anesthesiologist, and difficult if there was no chest rise and absent capnograph or peripheral oxygen saturation dropped to $90 \%$ or less despite the use of airway adjuncts (oral or nasal pharyngeal airway) or when there was the need for two providers to achieve mask ventilation [9]. If the resident could not mask ventilate the patient, it was the supervising consultant's decision when to intervene and take over. After three minutes of mask ventilation, the trachea was intubated using a Macintosh laryngoscope. The correct position of the endotracheal tube was confirmed by monitoring of end-tidal carbon dioxide and bilateral auscultation of the chest.

IBM Corp. Released 2010. IBM SPSS Statistics for Windows, Version 19.0. Armonk, NY: IBM Corp. was used for data analysis. Mean and standard deviation was used to express quantitative variables such as age, BMI, and STOP-Bang score. Qualitative observations such as gender, ASA status, STOP-Bang score ( $\geqslant 3 \&<3$ ), and difficult mask ventilation were presented as numbers and percentages. Chi-square test was used to observe the association between factors assessed and DMV and crude odds ratios were computed for each factor. Multivariate logistic stepwise regression analysis was employed to compute the adjusted odds ratio and significant contribution of the predictors. P-value $\leqslant 0.05$ was taken as significant. Accuracy was computed for STOP-Bang Score in predicting difficult mask ventilation. The area under the receiver operating characteristic curve (AUC-ROC) was used to determine the clinical value of the score. A value of 0.5 under the receiver operating characteristic (ROC) curve demonstrated that the performance of the variable was no better than chance, while a reading of 1.0 signified that the discrimination was perfect.

Sample size calculation was performed using MedCalc for Windows version 18.5 (MedCalc Software Ltd., Ostend, Belgium), taking into account the study performed by Corso et al., which reported an incidence of DMV of $23 \%$ with three or more risk factors signifying a high risk of OSA [2]. Five hundred and twelve patients were required to detect an improvement of discriminating power by the area under the curve (AUC) when $15 \%$ absolute difference (e.g., from $50 \%$ to $65 \%$ ) was considered clinically significant with $90 \%$ power and $1 \%$ type I error. Finally, a total of 530 patients were recruited for this study. 


\section{Cureus}

\section{Results}

This prospective, observational study included 530 patients. The mean age and BMI of the patients was 39.89 \pm 13.99 years and $27.135 \pm 5.54 \mathrm{~kg} / \mathrm{m} 2$ respectively. There were $302(57 \%)$ female and 228 (43\%) male patients. Regarding ASA status, 248 (46.8\%) belonged to ASA-I, 245 (46.2\%) to ASA-II and 37 (7\%) to ASAIII status.

Overall, DMV was found in 84 patients (15.8\%). Reasons/factors associated with DMV are provided in Table 1. Out of the 530 patients, $139(26.22 \%)$ had a STOP-Bang score of $\geqslant 3$, while $391(73.8 \%)$ had a score of $<3$. Among the 139 patients with a score of $\geqslant 3,55$ (39.5\%) were found to have DMV. Whereas, out of the 391 patients with a score of $<3$, only 29 patients $(7.5 \%)$ had DMV. This difference was statistically significant (P $\leqslant 0.001)$. Unadjusted odds ratio showed that all factors of the STOP-Bang criteria had two to eight times more likely association with DMV, while on multivariate analysis snoring, high blood pressure, BMI more than 35 $\mathrm{kg} / \mathrm{m} 2$, age more than 50 years, neck circumference more than $40 \mathrm{~cm}$ and male gender were significantly associated with DMV as presented in Table 1 .

\begin{tabular}{|c|c|c|c|c|c|c|}
\hline Variables & $\mathbf{N}$ & DMV n=84 (15.8\%) & P-Value & UOR (95\% Cl) & P-Value & aOR (95\% Cl) \\
\hline \multicolumn{7}{|l|}{ Snoring } \\
\hline Yes & 173 & $58(33.5 \%)$ & 0.0005 & $6.42(3.86-10.68)$ & 0.0005 & 5.32 (2.93-9.64) \\
\hline No & 357 & $26(7.3 \%)$ & & & & \\
\hline \multicolumn{7}{|c|}{ Tired during daytime } \\
\hline Yes & 96 & $25(26 \%)$ & 0.0005 & $2.24(1.31-3.81)$ & 0.687 & $1.16(0.56-2.39)$ \\
\hline No & 434 & 59 (13.6\%) & & & & \\
\hline \multicolumn{7}{|c|}{ Observed apnoea } \\
\hline Yes & 31 & 12 (38.7\%) & 0.0005 & 3.74 (1.74-8.05) & 0.550 & $1.40(0.46-4.25)$ \\
\hline No & 499 & $72(14.4 \%)$ & & & & \\
\hline \multicolumn{7}{|c|}{ High blood pressure } \\
\hline Yes & 167 & $47(28.1 \%)$ & 0.0005 & $3.45(2.14-5.57)$ & 0.007 & $2.32(1.26-4.27)$ \\
\hline No & 363 & $37(10.2 \%)$ & & & & \\
\hline \multicolumn{7}{|c|}{ BMI >35 kg } \\
\hline Yes & 45 & 24 (53.3\%) & 0.0005 & 8.09 (4.25-15.43) & 0.0005 & $4.62(2.11-10.08)$ \\
\hline No & 485 & $60(12.4 \%)$ & & & & \\
\hline \multicolumn{7}{|c|}{ Age $>50$ years } \\
\hline Yes & 125 & $42(33.6 \%)$ & 0.0005 & $4.37(2.68-7.14)$ & 0.0005 & $3.25(1.75-9.02)$ \\
\hline No & 405 & $42(10.4 \%)$ & & & & \\
\hline \multicolumn{7}{|c|}{ Neck circumference $>40 \mathrm{~cm}$} \\
\hline Yes & 43 & $19(44.2 \%)$ & 0.0005 & $5.14(2.67-9.91)$ & 0.003 & 3.42 (1.45-7.82) \\
\hline No & 487 & $65(13.3 \%)$ & & & & \\
\hline \multicolumn{7}{|c|}{ Gender Male } \\
\hline Yes & 218 & $46(21.1 \%)$ & 0.0005 & $1.93(1.21-3.08)$ & 0.021 & $2.03(1.11-3.72)$ \\
\hline No & 312 & $38(12.2 \%)$ & & & & \\
\hline
\end{tabular}

TABLE 1: Univariate and multivariable analysis showing association between STOP-Bang criteria and difficult mask ventilation $(n=530)$

DMV: difficult mask ventilation; UOR: unadjusted odds ratio; aOR: adjusted odds ratio; Cl: confidence interval 


\section{Cureus}

According to our results, the accuracy of STOP-Bang questionnaire in predicting difficult mask ventilation was $78.68 \%$ (95\% CI: 74.99-81.95), with a negative predictive value of $92.58 \%$ (95\% CI: 89.55-94.79). The sensitivity and specificity of the questionnaire in predicting DMV was found to be $65.48 \%$ and $81.17 \%$, respectively (Table 2). ROC plot in Figure 1 shows the AUC which is 0.82 (95\% CI: 0.77-0.87). The maximum sensitivity and minimum specificity was observed at the optimal cut-off point of STOP-Bang score of $\geqslant 3$.

\begin{tabular}{|c|c|c|}
\hline \multirow{2}{*}{ Parameter } & \multicolumn{2}{|c|}{ Stop Bang $\geq 3$} \\
\hline & Estimates & $(95 \% \mathrm{Cl})$ \\
\hline Sensitivity & $65.48 \%$ & $(54.83-74.77)$ \\
\hline Specificity & $81.17 \%$ & $(77.28-84.52)$ \\
\hline Positive Predictive Value & $39.57 \%$ & $(31.82-47.87)$ \\
\hline Negative Predictive Value & $92.58 \%$ & $(89.55-94.79)$ \\
\hline Diagnostic Accuracy & $78.68 \%$ & $(74.99-81.95)$ \\
\hline Cohen's kappa & 0.37 & $(0.28-0.45)$ \\
\hline
\end{tabular}

TABLE 2: Accuracy of STOP-Bang scores in predicting difficult mask ventilation

True Positive: 55; False Positive: 84; False Negative: 29; True Negative: 362; Cutoff: Stop Bang $\geq 3$; Prevalence of DMV: 15.8\% (84/530) 


\section{Cureus}

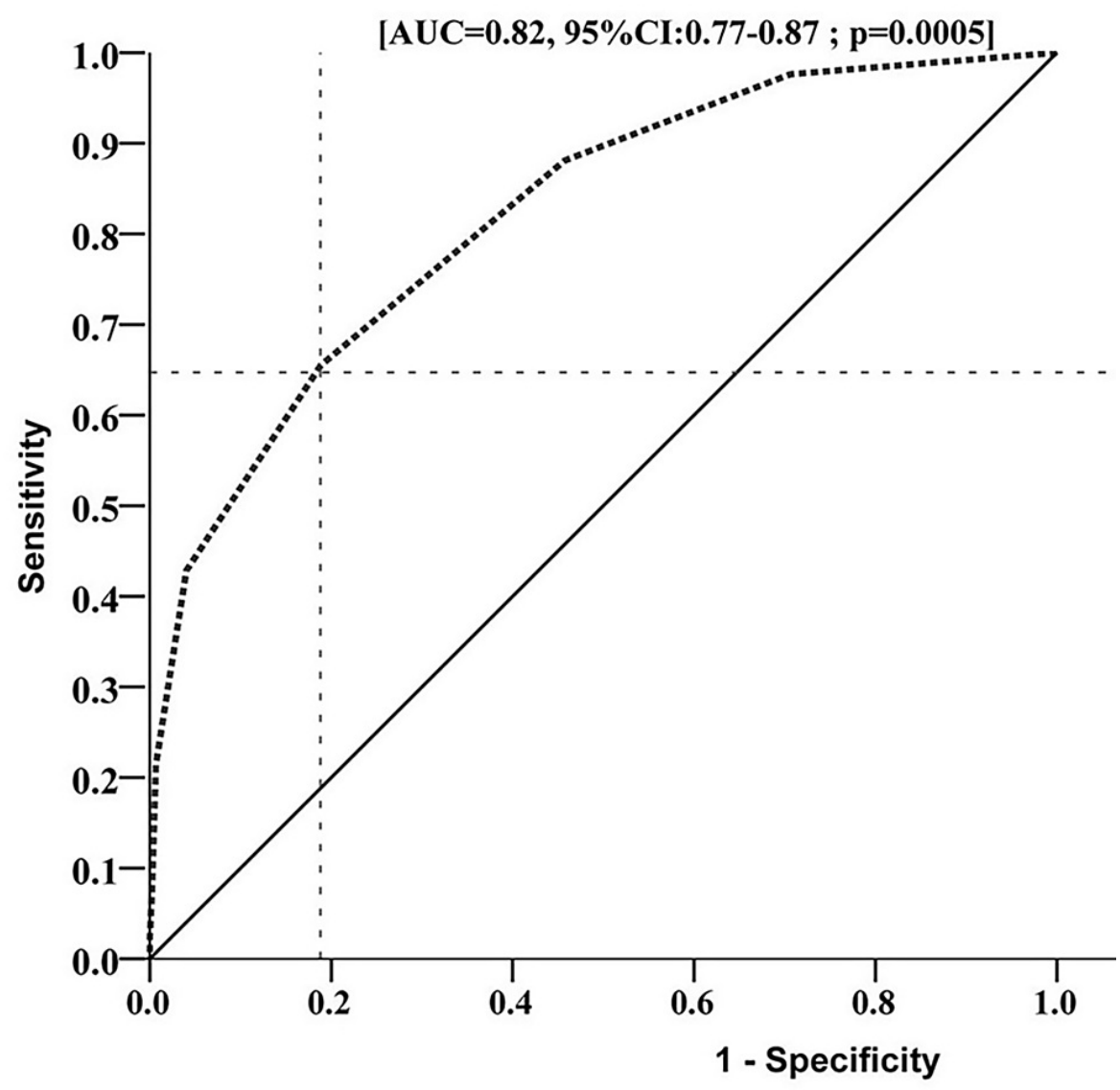

\section{FIGURE 1: Receiver operating characteristic (ROC) curve showing ability of STOP-Bang score in predicting difficult mask ventilation}

AUC: Area under the curve

\section{Discussion}

Mask ventilation is an essential skill that needs to be mastered by all anesthesiologists. It is an integral part of the majority of general anesthesia inductions and is a crucial technique to resort to for maintaining oxygenation during a failed or difficult intubation. In our prospective observational study on 530 patients, an overall frequency of DMV was found to be $15.8 \%$. Because of a lack of consensus on its definition, the reported incidence of DMV shows a wide disparity, ranging from $0.08 \%$ to $15 \%[3,4,10,11]$. Our result of $15.8 \%$ corresponds to the higher end of this range. A reason for this disparity might be the possible racial anatomical variations that can lead to DMV and difficult intubation in some populations [12]. Difficult or impossible mask ventilation has been found to be an important cause of permanent brain damage or even death during anesthesia [13]. Furthermore, it has been assessed that adult patients with DMV have a fourfold increased risk of difficult intubation [14]. Careful airway assessment and prediction of possible DMV are essential in devising a successful airway management plan to prevent adverse events and enhance patient safety. Thus, the anticipation of difficult airway and appropriate preparation can potentially decrease the serious consequences of hypoxia associated with failure of effective ventilation.

Our study showed that $39.5 \%$ of patients with a STOP-Bang score of $\geqslant 3$ had DMV compared to $7.5 \%$ in patients with a score of $<3$. In their study on 1399 patients, Cattano et al. endeavored to identify the predictive factors for DMV by conducting a retrospective subgroup analysis of patients' preoperative airway assessment before elective surgery [10]. According to their results, those with a history of OSA had a 17\% incidence of DMV. Our patients, in comparison, were not diagnosed OSA patients and our assessment was based on their STOP-Bang scores determined at preoperative assessment, which was not available in their retrospective analysis. The STOP-Bang questionnaire is a validated, easy-to-use tool to screen for OSA during the peri-operative period $[15,16]$. The scores obtained are used to identify the risk for OSA, presence of three or more criteria being considered an increased risk for OSA. Patients with OSA have episodes of upper airway obstruction during sleep with repeated arousals and desaturations [17]. Peri-operative respiratory complications are one of the most significant features of OSA that concerns the anesthesiologist 
and it has been identified as an independent predictor of difficult intubation [18]. DMV in patients with OSA may be explained by various factors, including tonsillar hyperplasia, a large tongue, pharyngeal wall collapse caused by redundant tissue, and increased neck circumference [11].

Out of the eight criteria of the STOP-Bang questionnaire, we found six criteria to be independently associated with a risk of DMV $(\mathrm{P}=0.0005)$, including snoring, high blood pressure, $\mathrm{BMI}>35 \mathrm{~kg} / \mathrm{m} 2$, age $>50$ years, neck circumference $>40 \mathrm{~cm}$ and male gender. The criteria identified by Cattano et al. as risk factors for DMV included age $\geqslant 47$ years, history of difficult intubation, BMI $\geqslant 35 \mathrm{~kg} / \mathrm{m} 2$, perceived short neck, neck circumference $\geqslant 40 \mathrm{~cm}$, presence of facial hair, and history of OSA [10]. Langerton et al., in their prospective study on 1502 patients identified five independent criteria for DMV including age > 55 years, BMI > 26 $\mathrm{kg} / \mathrm{m} 2$, a beard, absence of teeth, and history of snoring [19]. As we assessed only the STOP-Bang criteria, the presence of facial hair and the absence of teeth were not included in our data analysis.

It has been seen that approximately $24 \%$ of surgical patients are affected with OSA $[15,20]$. A large majority of patients having OSA remain undiagnosed [9]. For this reason, preoperative screening for OSA has been recommended by the Society of Anesthesia and Sleep Medicine and the American Society of Anesthesiologists [20,21]. High sensitivity for detection of OSA has been shown with a STOP-Bang score of $\geqslant 3$ [21]. However, the specificity is lower, resulting in a high false-negative rate. In their prospective observational trial including 22,660 anesthetics, Khetarpal et al. have reported that OSA is an independent predictor of difficult and impossible mask ventilation [3]. In our study, 139 (26.2\%) patients had a STOPBang score $\geqslant 3$. Out of these 139 patients, 55, (39.5\%) exhibited difficulty in mask ventilation. In contrast, Corso et al. have demonstrated an incidence of DMV of $23 \%$ in high OSA risk patients, but they used $\geqslant 5$ as the cut-off score to avoid false-negative results [2]. We used a score of $\geqslant 3$ for high risk of OSA as it has shown a high sensitivity and is recommended by the developers of the questionnaire [21]. Toshniwal et al. have reported that morbidly obese patients having a STOP-Bang score of $\geqslant 3$ demonstrated an increased risk for difficulty in airway management [8]. They also reported that muscle relaxation led to an improvement in mask ventilation in $43.9 \%$ of obese patients with DMV and $17 \%$ of these patients turned out to have difficult intubation as well. However, a STOP-Bang score of $<3$ does not completely rule out the possibility of encountering difficult mask ventilation. Although 391/530 patients in our study were low-risk for OSA (STOP-Bang < 3), 29 (7.42\%) of these patients had difficult mask ventilation.

In our study, the accuracy of the STOP-Bang questionnaire in predicting difficult mask ventilation was found to be $78.68 \%$ (95\% CI: 74.99-81.95), with a negative predictive value of $92.58 \%$ (95\% CI: 89.55-94.79). Although no direct comparison is available, our data show moderately high accuracy of STOP-Bang score for predicting DMV, while the negative predictive value is even higher. For a cut-off score of $\geqslant 3$, the sensitivity and specificity of the STOP-Bang questionnaire in predicting DMV was $65.48 \%$ and $81.17 \%$, respectively and the area under the ROC curve was 0.82 . This indicates that preoperative scoring of STOP-Bang criteria would be useful in predicting difficult mask ventilation and thus alerting the anesthesiologist towards taking appropriate safety measures. Although a lot of work has been done to identify criteria for predicting difficult intubation and practice guidelines and algorithms have been developed to reduce the incidence of potential adverse outcomes, similar work has not been done to identify factors that can predict DMV [19]. A very dangerous situation can develop if mask ventilation is inadequate in a patient in whom intubation turns out to be difficult or impossible. Therefore it is essential for patient safety to identify criteria that can alert the anesthesiologist towards the possibility of DMV.

OSA prevalence varies with the type of surgery, being highest among bariatric surgery patients [22]. Singh et al. in a cohort study on 819 patients demonstrated that anesthesiologists and surgeons neglected to identify many patients with pre-existing OSA prior to surgery [23]. It is crucial for anesthesiologists to assess patients undergoing surgery for OSA in order to devise an appropriate plan for the peri-operative care of such patients, owing to the mortality associated with it. Hiremath et al. demonstrated a strong association between diagnosed OSA and difficult intubation [24]. Plunkett et al have reported that, in their prospective study, nine out of ten patients with difficult mask ventilation at induction were proven to be suffering from OSA [25]. Due to differences in study designs, it is hard to compare our findings with those of the other authors. Some other studies also report that difficult mask ventilation was encountered more frequently in high-risk OSA patients, but either the STOP-Bang cut-off score used ( $\geqslant 5$ ) or the patient cohort (morbidly obese patients, diagnosed OSA patients, etc.) differed from our study population [2,7]. The hypothesis of our study was that the STOP-Bang questionnaire is useful in predicting DMV; however, our results demonstrate its high negative predictive value in ruling out DMV among surgical patients.

A limitation of this study is that other difficult airway predictors, such as Mallampati score, inter-incisor distance, and jaw movements were not analyzed, although they are evaluated in all surgical patients during a routine pre-anesthetic assessment at the authors' center. Evaluating these criteria, along with the STOPBang score during pre-anesthetic assessment would further enhance the value of STOP-Bang scores in the prediction of difficulty in airway management.

\section{Conclusions}

Our study has demonstrated that the STOP-Bang questionnaire has a high (92\%) negative predictive value for DMV. It can therefore be very helpful in ruling out the possibility of DMV. The questionnaire has been 
found to be $78 \%$ accurate in predicting DMV at the cut-off score of $\geqslant 3$. AUC was observed to be high due to the high rate of true negative values. We recommend the conduct of future studies with a higher cut-off STOP-Bang score and to combine it with other predictors of the difficult airway to assess its value in predicting DMV and to further consolidate our findings of its high negative predictive value.

\section{Additional Information \\ Disclosures}

Human subjects: Consent was obtained or waived by all participants in this study. Ethics Review Committee, Aga Khan University issued approval 4313-Ane-ERC-16. Approval for the study was granted by the institutional Ethics Review Committee. . Animal subjects: All authors have confirmed that this study did not involve animal subjects or tissue. Conflicts of interest: In compliance with the ICMJE uniform disclosure form, all authors declare the following: Payment/services info: All authors have declared that no financial support was received from any organization for the submitted work. Financial relationships: All authors have declared that they have no financial relationships at present or within the previous three years with any organizations that might have an interest in the submitted work. Other relationships: All authors have declared that there are no other relationships or activities that could appear to have influenced the submitted work.

\section{References}

1. Mathangi K, Mathews J, Mathangi CD: Assessment of perioperative difficult airway among undiagnosed obstructive sleep apnoea patients undergoing elective surgery: A prospective cohort study. Indian J Anaesth. 2018, 62:538-44. 10.4103/ija.IJA_158_18

2. Corso RM, Petrini F, Buccioli M, et al.: Clinical utility of preoperative screening with STOP-Bang questionnaire in elective surgery. Minerva Anestesiol. 2014, 80:877-84.

3. Kheterpal S, Han R, Tremper KK, Shanks A, Tait AR, O'Reilly M, Ludwig TA: Incidence and predictors of difficult and impossible mask ventilation. Anesthesiology. 2006, 105:885-91. 10.1097/00000542-20061100000007

4. Kheterpal S, Martin L, Shanks AM, Tremper KK: Prediction and outcomes of impossible mask ventilation: a review of 50,000 anesthetics. Anesthesiology. 2009, 110:891-7. 10.1097/ALN.0b013e31819b5b87

5. Raveendran R, Chung F: Ambulatory anesthesia for patients with sleep apnea. Ambulatory Anesthesia. 2015, 2:143-51.

6. Chung F, Abdullah HR, Liao P: STOP-Bang questionnaire: A practical approach to screen for obstructive sleep apnea. Chest. 2016, 149:631-8. 10.1378/chest.15-0903

7. Chung F, Yang Y, Brown R, Liao P: Alternative scoring models of STOP-bang questionnaire improve specificity to detect undiagnosed obstructive sleep apnea. J Clin Sleep Med. 2014, 10:951-8. $10.5664 /$ jcsm.4022

8. Toshniwal G, McKelvey GM, Wang H: STOP-Bang and prediction of difficult airway in obese patients . J Clin Anesth. 2014, 26:360-7. 10.1016/j.jclinane.2014.01.010

9. Gross JB, Apfelbaum JL, Caplan RA, et al.: Practice guidelines for the perioperative management of patients with obstructive sleep apnea: an updated report by the American Society of Anesthesiologists Task Force on Perioperative Management of patients with obstructive sleep apnea. Anesthesiology. 2014, 120:268-86. 10.1097/ALN.0000000000000053

10. Cattano D, Killoran PV, Cai C, Katsiampoura AD, Corso RM, Hagberg CA: Difficult mask ventilation in general surgical population: observation of risk factors and predictors. F1000Res. 2014, 3:204. 10.12688/f1000research.5131.1

11. El-Orbany M, Woehlck HJ: Difficult mask ventilation. Anesth Analg. 2009, 109:1870-80.

12. Prakash S, Kumar A, Bhandari S, Mullick P, Singh R, Gogia AR: Difficult laryngoscopy and intubation in the Indian population: an assessment of anatomical and clinical risk factors. Indian J Anaesth. 2013, 57:569-75. 10.4103/0019-5049.123329

13. Caplan RA, Posner KL, Ward RJ, Cheney FW: Adverse respiratory events in anesthesia: a closed claims analysis. Anesthesiology. 1990, 72:828-33. 10.1097/00000542-199005000-00010

14. Adnet F: Difficult mask ventilation: an underestimated aspect of the problem of the difficult airway? . Anesthesiology. 2000, 92:1217-8. 10.1097/00000542-200005000-00005

15. Young T, Evans L, Finn L, Palta M: Estimation of the clinically diagnosed proportion of sleep apnea syndrome in middle-aged men and women. Sleep. 1997, 20:705-6. 10.1093/sleep/20.9.705

16. Yang Y, Chung F: A screening tool of obstructive sleep apnea: STOP-Bang questionnaire . Sleep Med Clin. 2013, 8:65-72. 10.1016/j.jsmc.2012.11.004

17. Patil SP, Schneider H, Schwartz AR, Smith PL: Adult obstructive sleep apnea: pathophysiology and diagnosis. Chest. 2007, 132:325-37. 10.1378/chest.07-0040

18. Kheterpal S, Healy D, Aziz MF, et al.: Incidence, predictors, and outcome of difficult mask ventilation combined with difficult laryngoscopy: a report from the multicenter perioperative outcomes group. Anesthesiology. 2013, 119:1360-9. 10.1097/ALN.0000435832.39353.20

19. Langeron O, Masso E, Huraux C, Guggiari M, Bianchi A, Coriat P, Riou B: Prediction of difficult mask ventilation. Anesthesiology. 2000, 92:1229-36. 10.1097/00000542-200005000-00009

20. Chung F, Memtsoudis SG, Ramachandran SK, et al.: Society of anesthesia and sleep medicine guidelines on preoperative screening and assessment of adult patients with obstructive sleep apnea. Anesth Analg. 2016, 123:452-73. 10.1213/ANE.0000000000001416

21. Chung F, Yegneswaran B, Liao P, et al.: STOP questionnaire: a tool to screen patients for obstructive sleep apnea. Anesthesiology. 2008, 108:812-21. 10.1097/ALN.0b013e31816d83e4

22. Obstructive sleep apnoea and anaesthesia. Tutorial of the week 152. . (2009). Accessed: June 10, 2021: 


\section{Cureus}

https://resources.wfsahq.org/atotw/obstructive-sleep-apnoea-and-anaesthesia-tutorial-of-the-week-152/..

23. Singh M, Liao P, Kobah S, Wijeysundera DN, Shapiro C, Chung F: Proportion of surgical patients with undiagnosed obstructive sleep apnoea. Br J Anaesth. 2013, 110:629-36. 10.1093/bja/aes465

24. Hiremath AS, Hillman DR, James AL, Noffsinger WJ, Platt PR, Singer SL: Relationship between difficult tracheal intubation and obstructive sleep apnoea. Br J Anaesth. 1998, 80:606-11. 10.1093/bja/80.5.606

25. Plunkett AR, McLean BC, Brooks D, Plunkett MT, Mikita JA: Does difficult mask ventilation predict obstructive sleep apnea? A prospective pilot study to identify the prevalence of osa in patients with difficult mask ventilation under general anesthesia. J Clin Sleep Med. 2011, 7:473-7. 10.5664/JCSM.1310 